\title{
A Use of Assignment Sheet with Image Processing Technology Based on MFC
}

\author{
Ying'ang Zhang*, Ji Sun, Ran Zhu and Fan Zhang \\ School of Electrical Engineering and Automation, Nanjing Normal University, Nanjing 210042, China \\ *Corresponding author
}

\begin{abstract}
To solve the problem of the inconvenience of the work correction traditionally, the author designed an electronic assignments correcting system based on traditional homework paper pattern. According to image processing technology based on MFC, it realizes the functions of the image recognition, partition of papers and new files' name. The research shows that this design can complete the system's function requirements efficiently and reliably.
\end{abstract}

Keywords-C++ language; MFC library; digital image processing technology; assignment correcting system

\section{INTRODUCTION}

In teachers' work, there are lots of homework to correct. How to correct conveniently and how to store and manage students' homework need new methods and technologies to improve and innovate. As the rapid development of computer technology, network technology and image processing technology, the improvement and innovation will come true. We have designed an electronic assignment correcting system based on traditional assignment pattern, which,on the one hand, can make teachers correct assignments anytime conveniently. On the other hand, it can overcome the abuse of totally electronic homework. We find many subjects can't apply to electronic homework totally according to many practices in the past, such as mathematics and physics, because there will be lots of signs, signals and figures that is hard to describe online. So using papers and pens is the best choice. To sum up, it is a design combined traditional correcting with electronic computer technology, which means that students answer the questions on the paper and teachers correct assignments by computers and all kinds of terminals. Compared with totally electronization, the system can suit more kinds of subjects.

This design is mainly made up of several key elements which are 1. Dedicated papers, 2 The data base, 3 Image processing to the homework. The general work process is as show in figure 1.

It is not hard to see that the function of this system is that, according to the scan of the dedicated paper, we can realize the segmentation to the image of the paper, and then store the image after segmenting and the information of the two QR code and the date into the data base, so that these images can be called and discovered in the mobile terminal, which suits teachers very much.

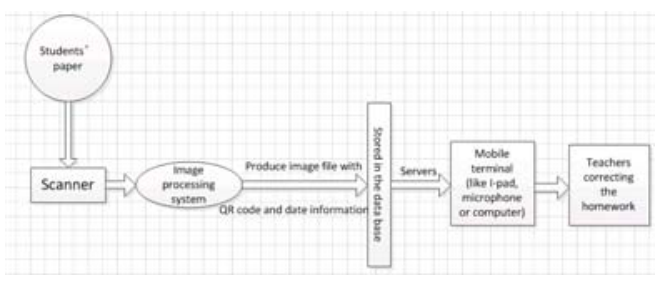

FIGURE I. GENERAL WORK PROCESS OF THE DESIGN

The assignment correcting system needs dedicated paper which is as shown in figure 2. The paper compromises two QR code, an date icon and text. Among them, the information of the first QR code is subject, and the second is student number. Students need wipe the blank of the day they do the homework, and write the very right title number in the blank of the text.

\section{IMAGe PRocessing OF ASSIGNMENT CORRECTING SYSTEM}

We can see this system is a big one, and this article mainly solves the part of image processing among the system. The framework of the subsystem is as shown in figure 3 .

The subsystem makes Visual Studio 2010 into design platform, and use $\mathrm{C}++$ as the programming language, and then build a new single document procedure with MFC library.

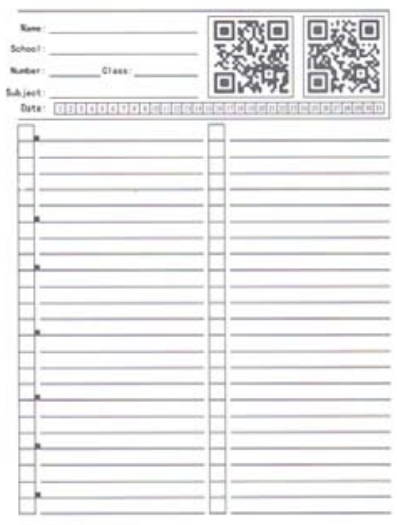

FIGURE II. DEDICATED PAPER FOR HOMEWORK 


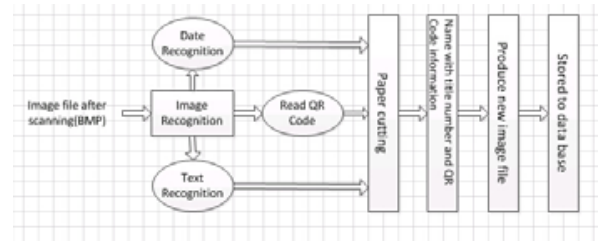

FIGURE III. BLOCK DIAGRAM OF IMAGE PROCESSING SYSTEM

First, put the students' paper of the homework into the scanner, and generate BMP according to the number of the resolution: 150dpi and then store the image into the same folder. Second, add file-header and info-header, read bitmap and cut the image into 4 parts: two QR code, date and text. Recognize QR code which is cut from the paper; handle the image of date by image binaryzation, so that we can identify the date according to comparing with the number of pixels. At the same time, use the same methods to cut small boxes on text, and after image binaryzation, recognize the title number and cut titles by these numbers which will be stored later. At last, name image files by $\mathrm{QR}$ code information, date information and title number of the text, and then store them into data base.

\section{ReAlization OF IMAge Processing System}

\section{A. How to Read and Cut Images}

Put papers into the scanner, adjust scanning parameters, and choose 150dpi as resolution ratio. The parameters are as shown in figure 4.

$\begin{array}{ll}\text { Property } & \text { Value } \\ \text { Image } & \\ \text { Dimensions } & 1264 \times 1752 \\ \text { Width } & 1264 \text { pixels } \\ \text { Height } & 1752 \text { pixels } \\ \text { Bit depth } & 24\end{array}$

FIGURE IV. IMAGE PARAMETERS

1) The bitmap loading

The bitmap file is made of three sections: bitmap-file header, bitmap information and the bitmap pixel data[1].

\section{a) Bitmap-file header}

Bitmap-file header is used for recognizing bitmap files. The structure of file-header of bitmap is as follows:

typedef struct tagBITMAPFILEHEADER \{

$\begin{array}{ll}\text { WORD } & \text { bfType; } \\ \text { DWORD } & \text { bfSize; } \\ \text { WORD } & \text { bfReserved1; } \\ \text { WORD } & \text { bfReserved2; } \\ \text { DWORD } & \text { bfOffBits; }\end{array}$

\section{\} BITMAPFILEHEADER;}

The value of bfType is "BM" $(0 \mathrm{x} 4 \mathrm{~d} 42)$, which means the file is bitmap. bfSize means the value of bitmap file.

\section{b) Bitmap information}

The value of the bitmap information is used for distributing internal storage, setting the palette, loading pixels and so on.[2] The structure of the bitmap information is as follows:

\section{Typedef struct tag BITMAPINFO \{ \\ BITMAPINFOHEADER bmiHeader;

RGBQUAD bmiColors; \\ \} BITMAPINFO;}

We can know that the bitmap information is made of twp sections: info-header and color table[1].

Info-header of bitmap includes number of bytes in single pixel, and it can reflect color format. Moreover, it includes the width and height of bitmap. The structure of bitmap-information header is as follows:

typedef struct tagBITMAPINFOHEADER \{

DWORD biSize;

LONG biWidth;

LONG biHeight;

WORD biPlanes;

WORD biBitCount;

DWORD biCompression;

DWORD biSizeImage;

LONG biXPelsPerMeter;

LONG biYPelsPerMeter;

DWORD biClrUsed;

DWORD biClrImportant;

\} BITMAPINFOHEADER;

biSize means numbers of bytes of BITMAPINFOHEADER, which is

sizeof(BITMAPINFOHEADER). biWith means image width in pixels.biHeight means image height in pixels. biPlanes shows number of bit planes of target device. biBitCount means the number of bytes in every pixel. biCompression is image compression format(the value is almost 0), biSizeImage means the value of image data in bytes[3].

\section{c) Bitmap data}

At last, the main section of bitmap under bitmap-file header, bitmap-information header and bitmap color table is bitmap data.[5] According to the different bitmap, the number of bytes occupied by the bitmap data are also different. For example, in an 8-bit bitmap, every byte represents one pixel,but two bytes represents one pixel in a 16-bit bitmap. Also, three bytes represents one pixel in a 24-bit bitmap while four bytes represents one pixel in a 32-bit bitmap[4].

\section{2) The bitmap cutting}

The aim of cutting in this design is to cut two QR code image, a date image and a image of text. Because we need 
rectangle structure to images, cutting arithmetic is convenient comparatively.

One cutting image needs four data items which is the left item, the lower item, the right item and the upper item. If we assume the coordinates of the lower-left corner is $(0,0)$, we will get the point that the value of the right item is more than the left one, as the upper one more than the lower one. Assume that bi. biWidth is the difference-value between the right item and the left one and bi. biHeight is the difference-value between the upper item and the lower one, so the pixel value of cutting paper is

biSizeImage $=($ bi.biWidth $*$ biBitCount +31$) / 32 * 4 *$ bi.biHeight,

Add new images' bitmap-file header:

bf.bfSize $=$ bf.bfOffBits + bi.biSizeImage, data:

Add new images' bitmap-information header and pixel BYTE* lpbuf; //pixel data

BYTE *Targetbuf

(BYTE*)HeapAlloc(GetProcessHeap(), 0, bi.biSizeImage);

for (int $\mathrm{i}=0$; $\mathrm{i}<$ bi.biHeight; $\mathrm{i}++$ )

\{

for (int $\mathrm{j}=0 ; \mathrm{j}<($ bi.biWidth $*$ bi.biBitCount $/ 8) ; \mathrm{j}++$ ) \{

Targetbuf $[$ (bi.biWidth $*$ bi.biBitCount $/ 8) * i$ $+\mathrm{j}]=\operatorname{lpbuf}[(\mathrm{i}+$ target.bottom $) *($ width $*$ bi.biBitCount $/ 8)+\mathrm{j}$ + target.left * bi.biBitCount / 8];

One point needs to be payed attention that the difference-value of the right item and the left one must be a multiple of 4 when we determine the coordinates.

When the coordinates are chosen are as follows:

typedef struct rect \{int left;int bottom;int right;int top;\}

rect;

rect test $1=\{42,111,1166,1290\}$;

$/ /$ text

coordinates

rect test $2=\{42,1300,1142,1350\} ;$ coordinates

rect test $3=\{882,1350,1142,1606\} ; \quad / /$ the first $Q R$ code coordinates

rect test $4=\{606,1350,874,1606\}$; code coordinates

We can acquire images as shown in figure 5,6.7 and 8 .

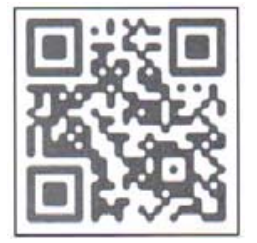

FIGURE V. THE FIRST QR CODE

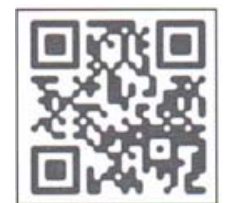

FIGURE VI. THE SECOND QR CODE

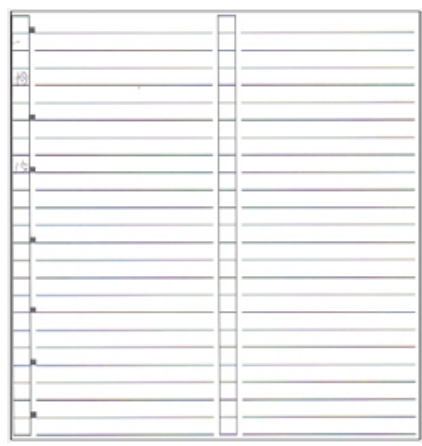

FIGURE VII. THE HOMEWORK AREA

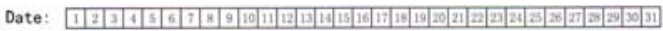

FIGURE VIII. THE DATE

\section{B. The QR Code Recognition in The Bitmap}

It is very convenient to recognize $\mathrm{QR}$ code that use $\mathrm{C}++$ language program library named LIBQRENCODE, and then run the program and input the path of the two QR code image.

\section{Gray-scale Image Processing}

In computer graphics and image processing, digital image gray scale is the basis of image recognition and processing. We often need to read digital image gray scale, and then carries on the analysis and processing, such as generating gray-scale histogram, gray-scale correction, image feature extracting, image sharpening, etc.

Digital images exist in the form of BITMAP, bitmap is a rectangular lattice, and pixel is the basic unit of the digital image. An $m * n$ size of the image is ranged from $m * n$ pixels. The degree of the light and shake of each pixel is identified by gray level among digital images. Generally we define the gray level of white 255 , the gray level of black as 0 , and by the contrast between black to white is evenly divided into 256 level. For black and white images, each pixel is said by one byte, while each pixel is said by three bytes in color images. Color images can be decomposed three monochromatic images which is red, green and blue, and these three kinds of color can compose any kinds of color. Therefore, in image processing, color image processing is usually based on its 
three monochromatic images processing respectively.

There is a gray-scale formula which is gray level $=\mathrm{r} * 0.114+\mathrm{g} * 0.587+\mathrm{b} * 0.299$ that can get gray level of every pixel point. Then, assume gray level $=\mathrm{r}=\mathrm{g}=\mathrm{b}$, so that we have accomplished gray-scale processing.

\section{The Date Recognition in The Bitmap}

There are 31 cells in the date image, and the upper and lower coordinates are as the same, which is that the lower value is 1309 and the upper value is 1343 . The coordinate of the left value of the first cell is 156 , and the difference-value between the right value and the left one in every cell is 32 .

Call the cutting function so that we can cut these 31 cells, and then do the gray-scale processing to them. After acquiring the number of black pixel points in every cell, we could get the date number according to find the most black pixel points.

\section{E. The Text Recognition in the Bitmap}

Like the date recognition, the text recognition needs cut all of the cells of the title number, and the cells' number is 48 . The coordinates of the front 24 cells are the same, which is that the left coordinate is 47 , the right 96 . The difference-value between the upper coordinate and the lower one in every cell is 48 , and the first cell's upper coordinate is 1276 . In a similar way, the behind 24 cells' left coordinate is 611 , right 659 . Cut them and write the number 1-48. Do the gray-scale processing and define threshold. The number of the black pixels which is written the title number is more than the number of the black pixels which is empty. After repeated experiments, the threshold is set to 50 .

Find the cells' number and testing sequence, and put them into an array, so we can calculate the upper and lower coordinates. The first cell's upper coordinate is 1274, and the last cell's lower coordinate is 126. Assume adjacent two topics' cell number is a and $b$. When the consequence is that $\mathrm{a}<\mathrm{b}<24$, the upper coordinate of $\mathrm{a}$ is

The lower one is

$$
\text { Top=1276- }(\mathrm{a}-1) * 48
$$

$$
\text { Bottom=1276- }(\mathrm{b}-1) * 48
$$

So we can cut the image of the topic whose title number is a.

When $\mathrm{a}<24<\mathrm{b}$, it means that the topic whose title number is a is divided two parts, and the left part's upper coordinate is

$$
\text { Top=1276- }(\mathrm{a}-1) * 48 \text {, }
$$

and the lower one is 126 . The right part's upper coordinate is 1276 , and the lower one is

$$
\text { Bottom=1276- }(\mathrm{b}-24-1) * 48 \text {, }
$$

So we can cut the two images of the topic whose title number is a.

When $24<\mathrm{a}<\mathrm{b}$, it means that the topic whose title number is a is located at the right part, and the upper coordinate is

and the lower coordinate is
Bottom=1276- $(\mathrm{b}-24-1) * 48$

We know that the last topic's lower coordinate is 126 . Assume that the last topic's cell number is $\mathrm{c}$. When $\mathrm{c}<24$, it means that the last topic has two parts, and the left part's upper coordinate is:

$$
\text { Top=1276- (c-1 )*48, }
$$

and the lower coordinate is 126 . The right part is the whole right part of the text, which means the upper coordinate is 1276 and the lower coordinate is 126 . When $\mathrm{c}>24$, it means the last topic is on the right, so the upper coordinate is

And the lower one is 126 .

$$
\text { Top=1276- }(\mathrm{c}-24-1) * 48 \text {, }
$$

When these sub functions are all done, name the complished text image as" QR code1-QR code2-date-topic number.BMP" and store them into the same folder. At last store them into data base.

\section{VALIDATION OF THE DESIGN}

The sample test image of the dedicated paper is as shown in figure 9 .

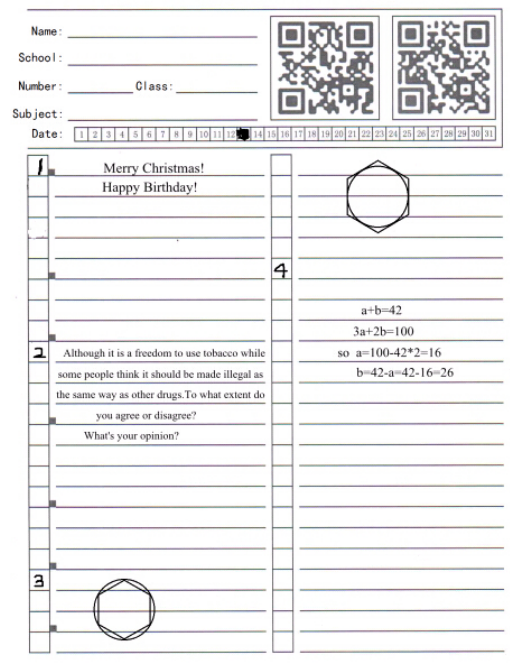

FIGURE IX. SAMPLE TEST IMAGE

After the image processing system, we have acquired 7 image files, which is as shown in figure 10. And the thumbnails of the images are as shown in figure 11. Topic number 3 has two files. The information of the first QR code is 9876543210987654321, and the information of the second QR code is 123456789012345678901234567890 . The date information is 13 .

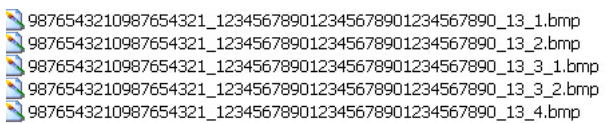

FIGURE X. NAMES OF THE IMAGES 


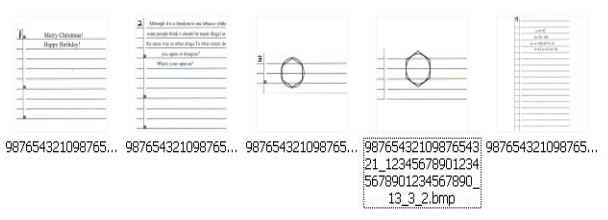

FIGURE XI. THUMBNAILS OF THE IMAGE FILES

\section{CONCLUSION}

To analyze the dedicated paper, the thesis has introduced complete design scheme. Start from scanning papers, we have done many things such as images' loading and cutting, images' gray-scale processing, QR codes recognition, date recognition and text recognition and cutting. The thesis has high success rate according to many tests, and the success rate is about $90 \%$, so we have completed all steps of image processing before storing the data base. Moreover, we have realized one-key processing, and teachers can reduce the time of loading the program.

This system also has good portability and augmentability, and it is convenient to add functions to this system.

\section{REFERENCES}

[1] Li Zhen, Yuan Dong, and Jiangzhou He, "C++ programming language (the 4th edition)," Tsinghua university publishing house, pp. 529-551, July 2010 .

[2] Rafael C.Gonzalez \& Richard E. Woods, “ Digital Image Processing”, Publishing House of Electronics Industry,pp. 63-81, December 2009.

[3] Dongjian He,'Digital Image Processing Technique(Second Edition)”,Xi'an electronic science and technology university publishing house,pp.145-178, May 2008.

[4] Zhiling Yang, Kai Wang,"Visual C++ Digital Image Acquiring,Processing And Use", Posts and Telecom Press,pp.335-336,January 2003.

[5] Hui Xu,"Visual C++ Digital Image Practical Engineering Case Selection", Posts and telecom press, pp.125-130. March 2004. 